\title{
Lung function and associations with multiple dimensions of dental health: a prospective observational cross-sectional study
}

\author{
Christian Henke ${ }^{1}$, Stephan Budweiser ${ }^{2}$ and Rudolf A. Jörres ${ }^{3^{*}}$
}

\begin{abstract}
Background: Epidemiological data suggest an association between respiratory diseases and periodontal health. However, the link between the overall dental status and single lung function measures, within a practical clinical context, is not well studied.
\end{abstract}

Methods: Following a prospective cross-sectional design, consecutive adult patients were evaluated. Next to spirometry, anthropometric data, profession, smoking status, symptoms, self-rated exercise performance, comorbidities, allergies and medication were determined. Assessment of dental status comprised carious lesions, dental fillings, missing teeth, dentures, insufficient fillings/dentures, implants, oral mucosa diseases, calculus, decayed-missed-filling-teeth (DMF-T)-index, periodontal screening-index, and orthopantomograms.

Results: Among 587 adult patients considered, 206 were included (119 female; median age 42.0 years; $56 \%$ smoking history). Most patients had dental fillings ( $86.9 \%)$, fix/mobile dentures $(66.5 \%)$, missing teeth $(56.8 \%)$ and calculus (84.0\%), the overall DMF-T being 15 (9; 21). Periodontitis was present in $53.9 \%$, an abnormal orthopanthomogram in $47.9 \%$ of subjects. Regarding spirometric indices expressed as \% predicted, dentures, missing teeth, oral mucosal diseases and a DMF-T > 15 (median) were associated with lower maximal expiratory flows at $25 \%$ of vital capacity $\left(\mathrm{MEF}_{25}\right)\left(\mathrm{p}<0.05\right.$ each). In adjusted logistic regression analyses, only dentures were associated with low $\mathrm{MEF}_{25} \%$ predicted and with the ratio of forced expiratory volume in $1 \mathrm{~s}$ to forced vital capacity (FEV $/$ /FVC; $p<0.05$ each). However, periodontitis and DMF-T were linked to age $(p<0.001)$ and packyears $(p<0.05)$ only.

Conclusion: Within a real-life clinical setting, only the presence of dentures showed weak associations with lung function, suggesting small airways dysfunction and obstruction. Most of the associations were explained by smoking habits and age.

Keywords: Periodontal disease, Oral health, Periodontitis, Lung disease, COPD

\section{Background}

Chronic obstructive pulmonary disease (COPD) is one of the most important chronic diseases worldwide [1], with a prevalence of about $5 \%$ to more than $20 \%$ in subjects aged $\geq 40$ years $[2,3]$. In addition to cigarette smoking, occupational exposures, indoor pollution, infections in early childhood, increasing age and low socioeconomic

\footnotetext{
*Correspondence: rudolf.joerres@med.uni-muenchen.de

${ }^{3}$ Institute and Outpatient Clinic for Occupational, Social

and Environmental Medicine, Ludwig-Maximilians-University, Munich, Germany

Full list of author information is available at the end of the article
}

status have been identified as important risk factors for COPD [4].

There is evidence for an association between oral health and systemic and/or chronic diseases, particularly cardiovascular diseases, diabetes, osteoporosis, but also respiratory diseases such as COPD or pneumonia $[5,6]$. Although difficult to prove, one hypothesis is that the oral cavity constitutes a potential source of pathogenetic micro-organisms that are transferred into the lower airways by micro-aspiration $[7,8]$. Moreover, in patients with periodontitis, inflammatory mediators or proteases from saliva or a release of cytokines into the circulation, 
evoked by inflammatory oral processes, might affect the lower respiratory system [5], in analogy to cardiovascular disorders [9].

These considerations render a link between oral health and especially COPD plausible. Systemic inflammation and recurrent exacerbations of chronic inflammation, often due to bacterial infections, are of major importance for the progression of COPD. Conversely, the presence of COPD and its associated frequent infections could impair oral health [8], but such interactions could be substantially confounded by socioeconomic status, lifestyle, education, dental care, medication and smoking habits.

As yet, most data refer to the association between COPD and periodontal disease [10-17]. These studies have provided conflicting results and used different techniques, outcome measures or populations for the assessment of periodontitis. If there should be a relevant link between dental health and lung function, it would be reasonable to expect that oral bacteria and systemic inflammation lead to impaired respiratory function prior to the manifestation of COPD. Additionally it could be hypothesized that lung function is associated with other dimensions of dental health, e.g. caries and endodontic lesions [8].

Many of the investigations were retrospective $[2-5,7$, $14,18,19]$ or differed with regard to study design and populations, methods and/or outcome measures used for dental assessment. Recently it has been reported that periodontal health (dental plaque, gingival bleeding and PD) was worse in smokers versus non-smokers, but in a multivariate model no major differences between smokers with versus without COPD occurred [18]. Similarly, in an earlier analysis of the NHANES-I population, no association was noted between any respiratory disease and the periodontal index [7]. In the larger NHANES III population comprising 7625 subjects, also no significant association between COPD and periodontitis has been found for former and non-smokers, except for current smokers [19].

Based on this it was concluded that the risk to develop periodontitis is predominately explained by smoking. In contrast, clinical attachment loss (CAL), PD, and number of missing teeth were found to be associated with reduced lung volumes, airflow limitation and hyperinflation but not with diffusing capacity [10]. Moreover, in very severe COPD, chronic marginal periodontitis was more frequent than in other severe respiratory diseases [14]. In line with this, CAL, PD and an oral hygiene test were higher in COPD patients as compared to a control population, after adjustment for covariates [11]. In a Chinese population plaque index was the most important periodontal factor related to COPD [12]. As a result, the most recent meta-analysis stated a significant association between periodontitis and COPD but also a substantial heterogeneity between studies [20]. Moreover, the funnel plot was markedly asymmetrical, which is considered to be indicative of a publication bias.

As a further relevant issue, the majority of studies focused on the presence of COPD as defined by a fixed $\mathrm{FEV}_{1} / \mathrm{FVC}<70 \%[11,12,19,21-23]$ or did not report lung function in detail $[7,14,24]$. In the present study, we covered all common spirometric lung function measures and did not restrict the analysis to the mere presence of COPD. We also explored the associations with dental health by using spirometric measures in absolute or $\%$ predicted values, applying established and, if possible, the novel GLI reference values. In particular we included $\mathrm{MEF}_{50}$ and $\mathrm{MEF}_{25}$ which are traditionally considered as being related to small airway dysfunction and indicative for the onset of obstructive lung disease. This was complemented by a comprehensive assessment of dental health as far as maximally feasible under the conditions of routine clinical practice.

We investigated the possible link between lung function and several dimensions of dental health beyond the periodontal status in a prospective "real-life" setting. For this, spirometry as well as a comprehensive, standardized dental examination by established techniques in dental practice were performed. We also included the decayedmissed-filled-teeth (DMFT)-index as an established score of dental health [25] which never has been explored regarding respiratory diseases in adults.

\section{Methods}

\section{Study Protocol}

Following the study protocol, consecutive, potentially eligible subjects consulting a general dental practice in Mühldorf, Bavaria, Germany, from July 2011 to January 2013 were asked for study participation. Inclusion criteria were age $\geq 18$ years and written informed consent. Subjects with known chronic infections (e.g. HIV or tuberculosis) or malignant diseases were excluded. We recorded anthropometric data and assessed medical history by routine dental exploration and a standardized questionnaire. Patients were asked for pulmonary symptoms (cough, sputum production, wheezing), comorbidities (pulmonary, cardiovascular, other), medication, the presence of type I allergies, smoking status (current, previous, packyears), the profession including possible workplace exposures, self-estimated exercise capacity, dyspnoea level, and previous periodontal treatment.

Spirometry (Masterscope/Labmanager; Carefusion, Höchberg, Germany) was performed according to the guidelines of the American Thoracic Society (ATS) [26] and evaluated using established [27] and, when possible, the novel global lungs initiative (GLI) reference values 
[28]. Forced expiratory volume in $1 \mathrm{~s}\left(\mathrm{FEV}_{1}\right)$, forced vital capacity (FVC), peak expiratory flow (PEF) and maximal expiratory flow rates at 50 and $25 \%\left(\mathrm{MEF}_{50}\right.$ and $\left.\mathrm{MEF}_{25}\right)$ of vital capacity were recorded. Clinically relevant bronchial obstruction was assumed at a ratio of $\mathrm{FEV}_{1} /$ FVC $<70 \%$ or, alternatively, <lower limit of normal (LLN) according to GLI. The spirometry was done by one single trained dental practice technician.

\section{Dental health assessment}

The comprehensive dental examination was also done by the same experienced dentist who performed the spirometry and who assessed the following items with regard to the presence and the number of respective teeth: carious lesions, dental fillings, missing teeth; dentures (fix, mobile, implants), insufficient fillings/dentures; presence of oral mucosal diseases (fistula, aphthous ulceration, gingivitis) and calculus. Finally, the DMFT-index was determined (with the highest value of 28 , as third molars are not considered) [25].

Periodontal status was evaluated by the periodontal screening-index (PSI) using an established WHO-probe (DB767R, PCP-11.5C, Aesculap, B. Braun, Melsungen, Germany) with a marker of length and a hemisphere at its end. The probe was introduced cautiously in four positions (mesiobuccal, distobuccal distopalatinal, mesiopalatinal) of each single tooth and probing depth (PD), bleeding and roughness of surface evaluated. Codes were chosen as follows [29]: (0) black marker (PD $3.5 \mathrm{~mm}$ ) completely apparent during probing, no calculus and/or insufficient edge of restauration occur, gums are healthy, no bleeding after probing; (1) black marker completely apparent during probing, no calculus and/or insufficient edge of restauration occur, minor bleeding after probing; (2) black marker completely apparent during probing, manifest calculus and/or insufficient edge of restauration occur; (3) black marker only partially apparent during probing, any other previously mentioned criteria may exist; (4) black marker disappearing completely during probing ( $\mathrm{PD}>5.5 \mathrm{~mm}$ ).

For documentation the maxilla and mandible was divided in sextants: maxillary (S1-S3, right-posterior, front, left-posterior) and mandibular (S4-S6, left-posterior, front, right-posterior), and the highest value was registered for each sextant. Periodontal status was categorized as follows: 0 in all sextants: no periodontitis; 1 or 2: no significant periodontitis but gingivitis; 3 : moderate periodontitis; 4 : severe periodontitis. The presence of periodontitis was defined according the following criteria: PSI $\geq 3$ or previous treatment of periodontitis, or bone loss $\geq 1$.

X-rays (orthopanthomogram), if available or required for medical reasons (e.g. dental surgery, search for apical lesions/dental focal points), were evaluated with regard to apical lesions, exposed furcation, displaced teeth, and horizontal loss of alveolar bone. The horizontal loss of alveolar bone was categorized as follows: grade 1 : bone loss $<1 / 3$ of alveolar bone; grade 2: bone loss $>1 / 3$ but $<2 / 3$, or grade 3 : bone loss $>2 / 3$ of alveolar bone. Following the study protocol, approved by the ethics committee of the Ludwig-Maximilians-University, Munich, Germany (study number 066/11), no X-rays were taken only for the study. Only if there was a medical reason for the comprehensive dental examination, new X-rays were taken.

\section{Statistical analyses}

Data were continuously documented and finally analyzed with SPSS software (IBM, Version 19.0, Chicago, IL, USA). Due to skewed distributions (Shapiro-Wilk Test) continuous variables were summarized as median values (quartiles), while using the non-parametric MannWhitney $U$ test to assess intergroup differences. In case of categorical variables the Fishers exact test was applied (two-sided). Bivariate correlation analyses (Pearson or Spearman) were employed to assess associations between spirometric measures and patients' characteristics. For the identification of independent associations with dental health, a logistic (dichotomous variables) or multivariate linear regression analysis was performed, whereby the single dimensions of dental health were used as dependent variables. Smoking, parameters being significant regarding the respective dimension of dental health, and single lung function indices (one by one) were introduced as explanatory variables. When using \% predicted values of lung function we did not additionally introduce age, gender and height, as these were already taken into account by the calculation of $\%$ predicted values. $\mathrm{P}$ values $<0.05$ were considered statistically significant.

\section{Results \\ Study population}

Among the 578 adult patients asked for study participation, 206 were included; 372 patients refused participation, delivered insufficient spirometry, or were excluded according to the study protocol. Anthropometric data, comorbidities, profession, job-related exposures, smoking history, respiratory symptoms, and exercise capability are shown in Table 1 . Regarding medication, most often prescribed were thyroxine/iodine $(\mathrm{n}=19 ; 9.2 \%)$, angiotensin-converting enzyme inhibitors and $\beta$-blockers (each $\mathrm{n}=7 ; 3.4 \%$ ).

\section{Lung function}

Overall, subjects showed a $\mathrm{FEV}_{1}$ of $96.0(87.6 ; 105.5) \%$ predicted (GLI), a FVC of $100.0(91.1 ; 108.9) \%$ predicted 
Table 1 Patients' characteristics of the total population ( $\mathrm{n}=\mathbf{2 0 6}$ )

\begin{tabular}{lc}
\hline Parameter & \\
\hline Age (years) & $42.0(32.0 ; 54.7)$ \\
BMI $\left(\mathrm{kg} / \mathrm{m}^{2}\right)$ & $24.6(21.8 ; 27.6)$ \\
Gender $(\mathrm{f})$ & $119(57.8 \%)$ \\
Current smoking & $59(28.6 \%)$ \\
Previous smoking & $58(28.2 \%)$ \\
History of smoking & $117(56.8 \%)$ \\
Pack years & $10.0(4.0 ; 20.7)$ \\
Cough & $28(13.6 \%)$ \\
Sputum production & $11(5.3 \%)$ \\
Wheezing & $4(1.9 \%)$ \\
Reduced performance & $45(21.8 \%)$ \\
NYHA class ( $\geq$ l) & $11(5.3 \%)$ \\
Asthma (self-reported) & $21(10.2 \%)$ \\
Systemic hypertension & $20(9.7 \%)$ \\
Cardiovascular disease & $9(4.4 \%)$ \\
Diabetes & $5(2.4 \%)$ \\
Hypothyreosis & $19(9.2 \%)$ \\
Osteoporosis & $4(1.9 \%)$ \\
Allergy type I & $42(20.2 \%)$ \\
Academic profession & $23(11.2 \%)$ \\
Job-related exposure & $27(13.1 \%)$ \\
\hline Resuls ar shown either as $(\%)$ or &
\end{tabular}

Results are shown either as $\mathrm{n}$ (\%) or median (quartiles)

$B M I$ body-mass-index; NYHA New York Heart Association

(GLI) and a ratio of $\mathrm{FEV}_{1} / \mathrm{FVC} 78.9(73.9 ; 83.2) \%$ (Table 2).

Among the 206 patients, 27 (13.1\%) had $\mathrm{FEV}_{1} /$ FVC $<70 \%$ and $25(12.1 \%)<$ LLN. When applying the GOLD criteria, 17 (GLI 13) subjects (8.3\%, GLI $6.3 \%$ ) had COPD I, and 10 (GLI 14) (4.9\%, GLI $6.8 \%$ ) COPD II.

There were inverse correlations between the number of packyears and $\mathrm{FEV}_{1}(\mathrm{~L})(\mathrm{r}=-0.189 ; \mathrm{p}=0.007), \mathrm{FEV}_{1}$ (\% predicted, GLI) $(-0.176 ; \mathrm{p}=0.011), \mathrm{FEV}_{1} / \mathrm{FVC}(\%)$ $(\mathrm{r}=-0.309 ; \mathrm{p}<0.001), \mathrm{FEV}_{1} / \mathrm{FVC}(\%$ predicted, GLI) $(-0.221 ; \mathrm{p}=0.001), \mathrm{MEF}_{50}(\mathrm{~L})(\mathrm{r}=-0.241 ; \mathrm{p}=0.003)$, $\mathrm{MEF}_{50}$ (\% predicted) $(\mathrm{r}=-0.227 ; \mathrm{p}=0.005), \mathrm{MEF}_{25}$ (L) $(\mathrm{r}=-0.338 ; \mathrm{p}<0.001)$ and $\mathrm{MEF}_{25}$ (\% predicted) $(\mathrm{r}=-0.336 ; \mathrm{p}<0.001)$. Age was inversely correlated to absolute values of $\mathrm{FEV}_{1}(\mathrm{r}=-0.440)$, $\mathrm{FVC}(\mathrm{r}=-0.329)$, $\mathrm{FEV}_{1} / \mathrm{FVC}(\mathrm{r}=-0.307), \mathrm{MEF}_{50}(\mathrm{r}=-0.363), \mathrm{MEF}_{25}$ $(\mathrm{r}=-0.570 ; \mathrm{p}<0.001$ each), PEF $(-0.152 ; \mathrm{p}=0.030)$. Regarding \% predicted values only $\mathrm{MEF}_{50}(\mathrm{r}=-0.167$; $\mathrm{p}=0.041)$ and $\operatorname{MEF}_{25}(\mathrm{r}=-0.304 ; \mathrm{p}<0.001)$ were significant.

\section{Dental health}

Most patients had dental fillings (86.9\%), fix/mobile dentures $(66.5 \%)$, missing teeth $(56.8 \%)$ or calculus $(84.0 \%)$,
Table 2 Spirometric measures of the total population

\begin{tabular}{|c|c|c|}
\hline Parameter & $\mathrm{n}$ & Median (quartiles) \\
\hline $\mathrm{FEV}_{1}(\mathrm{~L})$ & 206 & $3.20(2.75 ; 3.82)$ \\
\hline $\mathrm{FEV}_{1}(\%$ predicted $)$ & 206 & $102.6(92.2 ; 112.4)$ \\
\hline $\mathrm{FEV}_{1}(\% \text { predicted })^{\mathrm{GLI}}$ & 206 & $96.0(87.6 ; 105.5)$ \\
\hline $\mathrm{FVC}(\mathrm{L})$ & 206 & $4.06(3.51 ; 4.98)$ \\
\hline FVC (\% predicted) & 206 & $109.9(100.8 ; 119.2)$ \\
\hline FVC (\% predicted $)^{G L I}$ & 206 & $100.0(91.1 ; 108.9)$ \\
\hline $\mathrm{FEV}_{1} / \mathrm{FVC}(\%)$ & 206 & $78.9(73.9 ; 83.2)$ \\
\hline $\mathrm{FEV}_{1} / \mathrm{FVC}(\% \text { predicted })^{\mathrm{GLI}}$ & 206 & $96.7(91.1 ; 101.8)$ \\
\hline PEF (L/s) & 206 & $6.98(5.95 ; 8.65)$ \\
\hline PEF (\% predicted) & 206 & $95.0(84.7 ; 107.1)$ \\
\hline $\mathrm{MEF}_{50}(\mathrm{~L} / \mathrm{s})$ & 150 & $3.22(2.51 ; 4.18)$ \\
\hline $\mathrm{MEF}_{50}(\%$ predicted $)$ & 150 & $71.3(58.7 ; 92.0)$ \\
\hline $\mathrm{MEF}_{25}(\mathrm{~L} / \mathrm{s})$ & 150 & $0.94(0.52 ; 1.34)$ \\
\hline $\mathrm{MEF}_{25}$ (\% predicted) & 150 & $50.0(31.6 ; 68.2)$ \\
\hline
\end{tabular}

GLI reference values according to Global Lungs Initiative [18]

FEV forced expiratory volume in $1 \mathrm{~s}$; FVC forced vital capacity; PEF peak expiratory flow; $M E F_{50}$ maximal expiratory flow at $50 \%$ of vital capacity. $M E F_{25}$ maximal expiratory flow at $25 \%$ of vital capacity

and $97.5 \%$ a DMFT-index $>0$; overall $(\mathrm{n}=206)$ it was 15 $(9 ; 21)$ (Table 3). Regarding PSI, 24 subjects (11.7 \%) had no periodontitis (index 0$), 32(15.5 \%)$ index $1,59(28.6 \%)$ index 2 (gingivitis only), 51 (24.8\%) index 3 (moderate periodontitis), and 39 (18.9\%) index 4 (severe periodontitis); one patient did not have any teeth. Thus, 90 (43.7 \%) showed a PSI-index of $\geq 3$, median $2.0(1.0 ; 3.0)$. Moreover, $59(28.6 \%)$ had previous periodontal treatment. Thus, periodontitis was present in 111 patients (53.9\%).

\section{Table 3 Basic dental status of the total population} ( $n=206)$

\begin{tabular}{lrll}
\hline Variable & \multicolumn{1}{c}{$\mathbf{n}$} & \% & Number of teeth $^{\mathbf{a}}$ \\
\hline Carious lesions & 82 & 39.8 & $1.5(1.0 ; 3.0)$ \\
Dental fillings & 179 & 86.9 & $7.0(4.0 ; 11.0)$ \\
Dentures (fix or mobile) & 137 & $66.5 \%$ & - \\
$\quad$ Dentures fix & 133 & $64.6 \%$ & $7.0(3.0 ; 12.0)$ \\
Dentures mobile & 26 & $12.6 \%$ & - \\
Implants & 22 & 10.7 & $1.0(1.0 ; 3.5)$ \\
Insufficient fillings/dentures & 57 & 27.7 & $2.0(1.0 ; 2.5)$ \\
Missing teeth & 117 & 56.8 & $3.0(2.0 ; 8.5)$ \\
Disease of oral mucosa (any) & 36 & 17.5 & - \\
$\quad$ Apthen & 3 & 1.5 & - \\
Fistula & 11 & 5.3 & - \\
Gingivitis & 30 & 14.6 & - \\
Calculus & 173 & 84.0 & - \\
DMF-T $(>0)$ & 201 & 97.6 & $15(9.5 ; 21)$ \\
\hline
\end{tabular}

DMF-T Decayed-Missed-Filled-Teeth-index

a Referring to subjects affected, presented as median (quartiles) 
Among those with orthopanthomograms ( $\mathrm{n}=167), 34$ (20.4 \%) showed exposed furcations, 34 (20.4 \%) apical lesions, and 69 (41.3\%) alveolar bone loss (53 patients grade I, 16 grade II), 39 participants had no X-ray or evaluable X-ray documents. Thus, radiologic abnormalities of any kind were observed in 80 subjects (47.9\%).

\section{Association between periodontitis and spirometry}

Subjects with periodontitis $(\mathrm{n}=111)$ had higher age, BMI, weight and more packyears (Table 4), but less typeI allergies. Moreover, the absolute values of $\mathrm{FEV}_{1}, \mathrm{FVC}$, $\mathrm{FEV}_{1} / \mathrm{FVC}$ and $\mathrm{MEF}_{25}$ were significantly lower (Table 5), while this was not the case for $\%$ predicted values.

Table 4 Patients' characteristics in relation to the presence of periodontitis

\begin{tabular}{|c|c|c|c|c|c|}
\hline \multirow{2}{*}{$\begin{array}{l}\text { Parameter } \\
\text { Age (years) }\end{array}$} & \multicolumn{2}{|c|}{ No periodontitis } & \multicolumn{2}{|c|}{ Periodontitis } & \multirow{2}{*}{$\begin{array}{l}\text { p value } \\
<0.001\end{array}$} \\
\hline & 95 & $32.7(25.2 ; 40.9)$ & 111 & $51.8(41.5 ; 61.6)$ & \\
\hline Height (m) & 95 & $1.70(164 ; 1.76)$ & 111 & $1.70(1.65 ; 1.79)$ & 0.379 \\
\hline Weight (kg) & 95 & $67(60 ; 82)$ & 111 & $76(63 ; 89)$ & 0.006 \\
\hline $\mathrm{BMI}\left(\mathrm{kg} / \mathrm{m}^{2}\right)$ & 95 & $23.7(21.0 ; 26.3)$ & 111 & $25.4(22.5 ; 29.0)$ & 0.006 \\
\hline Gender (f) & 95 & $61(64.2 \%)$ & 111 & $59(53.2 \%)$ & 0.163 \\
\hline Current smoking & 95 & $28(29.5 \%)$ & 111 & $31(27.9 \%)$ & 0.877 \\
\hline Previous smoking & 95 & $21(22.1 \%)$ & 111 & $37(33.3 \%)$ & 0.088 \\
\hline History of smoking & 95 & $49(51.6 \%)$ & 111 & $68(61.3 \%)$ & 0.204 \\
\hline Pack years & 95 & $0.3(0.0 ; 5.5)$ & 111 & $5.0(0.0 ; 18.8)$ & 0.002 \\
\hline Cough & 95 & $9(9.5 \%)$ & 111 & 19 (17.1\%) & 0.153 \\
\hline $\begin{array}{l}\text { Sputum produc- } \\
\text { tion }\end{array}$ & 95 & $3(3.2 \%)$ & 111 & $8(7.2 \%)$ & 0.230 \\
\hline Wheezing & 95 & $0(0.0 \%)$ & 111 & $4(3.6 \%)$ & 0.126 \\
\hline $\begin{array}{l}\text { Reduced perfor- } \\
\text { mance }\end{array}$ & 95 & 15 (15.8\%) & 111 & $30(27.0)$ & 0.063 \\
\hline NYHA class $(\geq 1)$ & 95 & $3(3.2 \%)$ & 111 & $8(7.2)$ & 0.230 \\
\hline $\begin{array}{l}\text { Asthma (self- } \\
\text { reported) }\end{array}$ & 95 & 10 (10.5 \%) & 111 & $11(9.9 \%)$ & 0.532 \\
\hline $\begin{array}{l}\mathrm{COPD}\left(\mathrm{FEV}_{1} /\right. \\
\text { FVC }<0.7)\end{array}$ & 95 & $9(9.5 \%)$ & 111 & $19(17.1 \%)$ & 0.153 \\
\hline $\begin{array}{l}\text { Systemic hyper- } \\
\text { tension }\end{array}$ & 95 & $5(5.3 \%)$ & 111 & $15(13.5 \%)$ & 0.059 \\
\hline $\begin{array}{l}\text { Cardiovascular } \\
\text { disease }\end{array}$ & 95 & $2(2.1 \%)$ & 111 & $7(6.3 \%)$ & 0.182 \\
\hline Diabetes & 95 & $1(1.1 \%)$ & 111 & $4(3.6 \%)$ & 0.377 \\
\hline Hypothyreosis & 95 & $7(7.4 \%)$ & 111 & 12 (10.8\%) & 0.473 \\
\hline Osteoporosis & 95 & $1(1.1 \%)$ & 111 & $3(2.7 \%)$ & 0.626 \\
\hline Allergy type I & 95 & $42(44.2 \%)$ & 111 & $29(26.1 \%)$ & 0.008 \\
\hline $\begin{array}{l}\text { Academic profes- } \\
\text { sion }\end{array}$ & 95 & $13(13.7 \%)$ & 111 & 10 (9.0\%) & 0.375 \\
\hline $\begin{array}{l}\text { Job-related expo- } \\
\text { sure }\end{array}$ & 95 & $13(13.7 \%)$ & 111 & $14(12.6 \%)$ & 0.490 \\
\hline $\begin{array}{l}\text { Defined by } \mathrm{PSI} \geq 3 \text { or } \\
\text { Italic characters are }\end{array}$ & & $\begin{array}{l}\text { s treatment of } p \\
\text { statistically sign }\end{array}$ & $\mathrm{dc}$ & $\begin{array}{l}\text { titis or alveolar bon } \\
\text { comparisons }(p<0 .\end{array}$ & $\begin{array}{l}\text { e loss } \geq 1 \\
.05)\end{array}$ \\
\hline $\begin{array}{l}\text { BMI body-mass-inde } \\
\text { obstructive pulmona } \\
\text { vital capacity }\end{array}$ & & New York Heart & Associa & tion; $C O P D$ chron & forced \\
\hline
\end{tabular}

In multivariate logistic regression analyses taking the presence of periodontitis as dependent variable together with the absolute value of $\mathrm{FEV}_{1}, \mathrm{FVC}, \mathrm{FEV}_{1} / \mathrm{FVC}$ or PEF (one by one), only age $(\mathrm{p}<0.001)$ and packyears $(\mathrm{p}<0.05)$ were statistically significant. When introducing the absolute value of $\mathrm{MEF}_{50} / \mathrm{MEF}_{25}$, only age remained significant $(\mathrm{p}<0.001)$. In multivariate analyses including $\%$ predicted values, only packyears $(\mathrm{p} \leq 0.01$ each) were significantly associated with periodontitis.

\section{Association between basic dental status including DMF-T and spirometry}

Regarding specific characteristics (Table 3), subjects with dental fillings $(\mathrm{n}=179)$ showed higher absolute values of $\mathrm{FEV}_{1}(\mathrm{p}=0.020), \operatorname{FVC}(\mathrm{p}=0.007)$, PEF $(\mathrm{p}=0.002)$, height and better exercise performance ( $<0.05$ each). Lung function indices as $\%$ predicted were not significantly different.

Patients with dentures had lower absolute values of $\mathrm{FEV}_{1}(\mathrm{p}<0.001), \mathrm{FVC}(\mathrm{p}=0.002), \mathrm{FEV}_{1} / \mathrm{FVC}, \mathrm{MEF}_{50}$, $\mathrm{MEF}_{25}$, and $\mathrm{MEF}_{25} \%$ predicted ( $\mathrm{p}<0.001$ each), but higher age $(p<0.001)$ and were more often current smokers $(\mathrm{p}=0.034)$.

Subjects with missing teeth $(\mathrm{n}=117)$ had lower absolute values for $\mathrm{FEV}_{1}(\mathrm{p}<0.001), \mathrm{FVC}(\mathrm{p}=0.001), \mathrm{FEV}_{1} /$

Table 5 Spirometric data in relation to the presence of periodontitis

\begin{tabular}{|c|c|c|c|c|c|}
\hline \multirow{2}{*}{$\begin{array}{l}\text { Parameter } \\
\mathrm{FEV}_{1}(\mathrm{~L})\end{array}$} & \multicolumn{2}{|c|}{ No periodontitis } & \multicolumn{2}{|c|}{ Periodontitis } & \multirow{2}{*}{$\begin{array}{l}\text { p value } \\
0.001\end{array}$} \\
\hline & 95 & $3.42(2.97 ; 3.88)$ & 111 & $3.05(2.53 ; 3.75)$ & \\
\hline $\begin{array}{l}\mathrm{FEV}_{1}(\% \text { pre- } \\
\text { dicted) }\end{array}$ & 95 & $\begin{array}{l}100.9(91.9 ; \\
112.7)\end{array}$ & 111 & $\begin{array}{l}102.8(92.3 ; \\
112.3)\end{array}$ & 0.580 \\
\hline $\begin{array}{l}\mathrm{FEV}_{1}(\% \\
\text { predicted })^{\mathrm{GLI}}\end{array}$ & 95 & $\begin{array}{l}95.9(88.9 ; \\
107.1)\end{array}$ & 111 & $\begin{array}{l}96.7(86.5 ; \\
105.3)\end{array}$ & 0.847 \\
\hline $\mathrm{FVC}(\mathrm{L})$ & 95 & $4.24(3.70 ; 5.04)$ & 111 & $3.88(3.41 ; 4.98)$ & 0.025 \\
\hline $\begin{array}{l}\text { FVC (\% pre- } \\
\text { dicted) }\end{array}$ & 95 & $\begin{array}{l}108.6(99.6 ; \\
118.6)\end{array}$ & 111 & $\begin{array}{l}110.9 \text { (101.9; } \\
121.0)\end{array}$ & 0.333 \\
\hline $\begin{array}{l}\mathrm{FVC}(\% \\
\text { predicted })^{\mathrm{GLI}}\end{array}$ & 95 & $\begin{array}{l}101.1(91.2 ; \\
110.4)\end{array}$ & 111 & $\begin{array}{l}99.6(90.3 ; \\
107.3)\end{array}$ & 0.384 \\
\hline $\mathrm{FEV}_{1} / \mathrm{FVC}(\%)$ & 95 & $79.9(76.0 ; 84.2)$ & 111 & $77.8(72.4 ; 82.2)$ & 0.008 \\
\hline $\begin{array}{l}\mathrm{FEV}_{1} / \mathrm{FVC}(\% \\
\text { predicted }^{\mathrm{GLI}}\end{array}$ & 95 & $\begin{array}{l}95.7(91.3 ; \\
100.0)\end{array}$ & 111 & $\begin{array}{l}97.6(90.9 ; \\
102.9)\end{array}$ & 0.263 \\
\hline PEF (L/s) & 95 & $7.01(6.18 ; 8.73)$ & 111 & $6.80(5.71 ; 8.61)$ & 0.252 \\
\hline $\begin{array}{l}\text { PEF (\% pre- } \\
\text { dicted) }\end{array}$ & 95 & $\begin{array}{l}94.3(83.5 \\
\text { 106.0) }\end{array}$ & 111 & $\begin{array}{l}95.0(85.7 \text {; } \\
109.2)\end{array}$ & 0.469 \\
\hline $\mathrm{MEF}_{50}(\mathrm{~L} / \mathrm{s})$ & 70 & $3.28(2.73 ; 4.22)$ & 80 & $3.12(2.37 ; 4.16)$ & 0.240 \\
\hline $\begin{array}{l}\mathrm{MEF}_{50}(\% \text { pre- } \\
\text { dicted) }\end{array}$ & 70 & $70.9(61.5 ; 92.7)$ & 80 & $74.3(53.6 ; 91.3)$ & 0.928 \\
\hline $\mathrm{MEF}_{25}(\mathrm{~L} / \mathrm{s})$ & 70 & $1.07(0.80 ; 1.50)$ & 80 & $0.84(0.44 ; 1.24)$ & 0.003 \\
\hline $\begin{array}{l}\mathrm{MEF}_{25}(\% \text { pre- } \\
\text { dicted) }\end{array}$ & 70 & $52.6(38.4 ; 73.0)$ & 80 & $46.5(28.8 ; 63.2)$ & 0.067 \\
\hline
\end{tabular}

Defined by $\mathrm{PSI} \geq 3$ or previous treatment of periodontitis or alveolar bone loss $\geq 1$ Italic characters are used for statistically significant comparisons $(p<0.05)$ See Table 1 
FVC $(\mathrm{p}=0.017), \mathrm{MEF}_{50}(\mathrm{p}=0.003), \mathrm{MEF}_{25}(\mathrm{p}<0.001)$ and $\mathrm{MEF}_{25} \%$ predicted $(\mathrm{p}=0.021)$, but higher age $(\mathrm{p}<0.001)$, BMI $(\mathrm{p}=0.024)$ and more packyears $(\mathrm{p}=0.008)$. Moreover they more often showed hypertension, hypothyreosis, cough, reduced exercise performance ( $\mathrm{p}<0.05$ each), and previous smokers history $(\mathrm{p}=0.009)$.

Subjects with oral mucosal diseases $(\mathrm{n}=36)$ had lower values of $\mathrm{MEF}_{25} \%$ predicted ( $\left.\mathrm{p}=0.025\right)$, more often male gender $(\mathrm{p}=0.005)$ and reduced exercise performance $(\mathrm{p}=0.028)$. Patients with calculus showed lower $\mathrm{FEV}_{1} / \mathrm{FVC}(\mathrm{p}=0.026)$, higher age $(\mathrm{p}=0.006)$, BMI $(\mathrm{p}=0.008)$, weight $(\mathrm{p}=0.021)$ and more often hypertension $(p=0.049)$. Lung function did not significantly differ between patients with versus without carious lesions, insufficient fillings/dentures and implants.

In adjusted bivariate logistic regression analyses dentures were significantly associated with $\mathrm{MEF}_{25} \%$ predicted (Table 6), and marginally failed statistical significance when also including age $(\mathrm{p}=0.067)$. No statistical associations with lung function indices (absolute or $\%$ predicted) were found in adjusted multivariate analyses including dental fillings, missing teeth, oral mucosal disease, calculus, carious lesions, insufficient fillings/dentures and implants.

Subjects with a DMF-T index $\geq 15$ (median; $\mathrm{n}=105$ ) versus those with lower values were characterized by a higher age $(\mathrm{p}<0.001)$, more packyears $(\mathrm{p}=0.001)$, more often systemic hypertension, previous smoking history, and impaired exercise performance ( $\mathrm{p}<0.05$ each). Regarding spirometry, the absolute values of $\mathrm{FEV}_{1}, \mathrm{FVC}$, $\mathrm{FEV}_{1} / \mathrm{FVC}, \mathrm{MEF}_{50}, \mathrm{MEF}_{25}$, and $\mathrm{MEF}_{25} \%$ predicted were significantly lower in patients with a DMF-T-index $\geq 15$ (Table 7).

In a bivariate logistic regression analysis, taking DMF-T index (either $<$ versus $\geq 15$ ) as dependent variable together with the absolute of $\mathrm{FEV}_{1}, \mathrm{FVC}, \mathrm{FEV}_{1} / \mathrm{FVC}$ or PEF, again only age $(\mathrm{p}<0.001)$ and packyears $(\mathrm{p}<0.05)$ were significant. When introducing absolute values of $\mathrm{MEF}_{50} / \mathrm{MEF}_{25}$, only age remained significant $(\mathrm{p}<0.001)$. In multivariate analyses including $\%$ predicted values, packyears were still significant ( $\mathrm{p} \leq 0.01$ each). In a multivariate regression analysis, taking DMF-T as dependent variable, similar results were obtained.

Table 6 Association of dentures and $\mathrm{MEF}_{25} \%$ predicted using bivariate regression analysis

\begin{tabular}{llll}
\hline Variable & Coefficient B & P value & Exp (B) $(\mathbf{9 5} \%$ CI) \\
\hline MEF $_{25} \%$ predicted & -0.029 & $<0.001$ & $0.972(0.958-0.986)$ \\
Current smoking & -1.165 & 0.004 & $0.312(0.140-0.693)$
\end{tabular}

Italic characters are used for statistically significant comparisons $(p<0.05)$ $M E F_{25}$ maximal expiratory flow at $25 \%$ of vital capacity, $\operatorname{Exp}(B)$ odds ratio
Table 7 Spirometric data in relation to the DMF-T index

\begin{tabular}{|c|c|c|c|c|c|}
\hline \multirow{2}{*}{$\begin{array}{l}\text { Parameter } \\
\mathrm{FEV}_{1}(\mathrm{~L})\end{array}$} & \multicolumn{2}{|c|}{ DMF-T $<15$} & \multicolumn{2}{|c|}{ DMFT $\geq 15$} & \multirow{2}{*}{$\begin{array}{l}\text { p value } \\
<0.001\end{array}$} \\
\hline & 101 & $3.43(3.03 ; 3.86)$ & 105 & $2.97(2.43 ; 3.76)$ & \\
\hline $\begin{array}{l}\mathrm{FEV}_{1}(\% \text { pre- } \\
\text { dicted) }\end{array}$ & 101 & $\begin{array}{l}100.9(92.9 ; \\
112.4)\end{array}$ & 105 & $\begin{array}{l}103.0(91.0 ; \\
112.7)\end{array}$ & 0.829 \\
\hline $\begin{array}{l}\mathrm{FEV}_{1}(\% \\
\text { predicted }^{\mathrm{GLI}}\end{array}$ & 101 & $\begin{array}{l}95.4(88.7 ; \\
105.4)\end{array}$ & 105 & $\begin{array}{l}97.1(86.4 ; \\
105.5)\end{array}$ & 1 \\
\hline $\mathrm{FVC}(\mathrm{L})$ & 101 & $4.24(3.81 ; 5.18)$ & 105 & $3.78(3.32 ; 4.83)$ & $<0.001$ \\
\hline $\begin{array}{l}\text { FVC (\% pre- } \\
\text { dicted) }\end{array}$ & 101 & $\begin{array}{l}\text { 109.0 (99.6; } \\
118.7)\end{array}$ & 105 & $\begin{array}{l}110.6(102.2 ; \\
121.3)\end{array}$ & 0.512 \\
\hline $\begin{array}{l}\text { FVC }(\% \\
\quad \text { predicted })^{G L I}\end{array}$ & 101 & $\begin{array}{l}\text { 101.3 (91.1; } \\
109.8)\end{array}$ & 105 & $\begin{array}{l}99.6(90.4 ; \\
108.2)\end{array}$ & 0.493 \\
\hline $\mathrm{FEV}_{1} / \mathrm{FVC}(\%)$ & 101 & $79.8(75.3 ; 84.2)$ & 105 & $77.8(73.0 ; 82.0)$ & 0.017 \\
\hline $\begin{array}{l}\mathrm{FEV}_{1} / \mathrm{FVC}(\% \\
\text { predicted }^{\mathrm{GLI}}\end{array}$ & 101 & $\begin{array}{l}95.6(91.3 ; \\
100.1)\end{array}$ & 105 & $\begin{array}{l}97.8(90.9 ; \\
102.5)\end{array}$ & 0.263 \\
\hline $\operatorname{PEF}(L / s)$ & 101 & $7.03(6.20 ; 8.89)$ & 105 & $6.74(5.73 ; 8.06)$ & 0.113 \\
\hline $\begin{array}{l}\text { PEF (\% pre- } \\
\text { dicted) }\end{array}$ & 101 & $\begin{array}{l}93.9(83.9 ; \\
105.4)\end{array}$ & 105 & $\begin{array}{l}95.1(85.3 ; \\
110.4)\end{array}$ & 0.246 \\
\hline $\mathrm{MEF}_{50}(\mathrm{~L} / \mathrm{s})$ & 75 & $3.38(2.83 ; 4.29)$ & 75 & $2.82(2.11 ; 4.06)$ & 0.012 \\
\hline $\begin{array}{l}\mathrm{MEF}_{50}(\% \text { pre- } \\
\text { dicted) }\end{array}$ & 75 & $71.6(62.4 ; 92.6)$ & 75 & $69.9(51.2 ; 91.3)$ & 0.382 \\
\hline $\mathrm{MEF}_{25}(\mathrm{~L} / \mathrm{s})$ & 75 & $1.10(0.81 ; 1.50)$ & 75 & $0.73(0.43 ; 1.15)$ & $<0.001$ \\
\hline $\begin{array}{l}\mathrm{MEF}_{25}(\% \text { pre- } \\
\text { dicted) }\end{array}$ & 75 & $55.1(39.8 ; 73.6)$ & 75 & $42.5(29.1 ; 62.2)$ & 0.014 \\
\hline
\end{tabular}

Comparing subjects with DMF-T index either $<$ versus $\geq 15$ (median)

Italic characters are used for statistically significant comparisons $(p<0.05)$

$D M F-T$ decayed-missed-filled-teeth-index, for other abbreviations see Table 1

\section{Association between abnormal radiologic findings and spirometry}

Subject with $(\mathrm{n}=80)$ versus without radiologic abnormalites those were more often males $(\mathrm{p}=0.011)$, had higher BMI $(\mathrm{p}=0.024)$, weight $(\mathrm{p}=0.006)$, age $(\mathrm{p}<0.001)$ and more packyears $(\mathrm{p}=0.010)$. Regarding spirometry, the absolute values of $\mathrm{FEV}_{1}, \mathrm{FEV}_{1} / \mathrm{FVC}$ and $\mathrm{MEF}_{25}$ were significantly $(\mathrm{p}<0.01$ each) lower in patients with abnormal radiologic findings.

In an adjusted bivariate logistic regression analysis, taking the presence of any radiologic finding as dependent variable, significant association were only detected for age ( $\mathrm{p}<0.001$ each) when including absolute values, or packyears $(\mathrm{p}<0.05$ each) when including \% predicted values.

\section{Association between abnormal dental health and the presence of COPD}

Patients with versus without $\mathrm{FEV}_{1} / \mathrm{FVC}<70 \%$ or $<\mathrm{LLN}$ did not differ with regard to periodontitis (Table 4), the DMF-T index or the other single dimensions of dental health. However, these patients more often were male, had more packyears and current smoking history ( $\mathrm{p}<0.05$ each). In adjusted multivariate analyses, $\mathrm{FEV}_{1} /$ FVC $<70 \%$ was significantly associated with the presence of dentures (Table 8). 
Table 8 Association of dentures and FEV $_{1} /$ FVC $<70 \%$ using bivariate regression analysis

\begin{tabular}{lcll}
\hline Variable & Coefficient B & p value & Exp (B) $(\mathbf{9 5} \%$ Cl) \\
\hline $\mathrm{FEV}_{1} / \mathrm{FVC}<70 \%$ & 1.208 & 0.026 & $3.346(1.156-9.687)$ \\
Current smoking & -0.862 & 0.011 & $0.422(0.217-0.821)$ \\
gender $(\mathrm{f} / \mathrm{m})$ & 0.153 & 0.627 & $1.166(0.628-2.164)$ \\
\hline
\end{tabular}

Italic characters are used for statistically significant comparisons $(p<0.05)$

$F E V$, forced expiratory volume in $1 \mathrm{~s}$; FVC forced vital capacity; Exp (B) odds ratio

The major results have been presented at the annual meeting of the European Respiratory Society 2014 [30].

\section{Discussion}

The present study is the first prospective cross-sectional study evaluating the associations between spirometric measures and multiple dimensions of dental health in a random population of adult patients from a general dentist's practice. Using \% predicted values, the associations of the different indices of dental health with lung function appeared to be rather weak. While patients with dentures, missing teeth, oral mucosal diseases and a higher DMF-T-index presented with lower $\mathrm{MEF}_{25}$, in adjusted multivariate analyses, only the presence of dentures was significantly associated with $\mathrm{MEF}_{25} \%$ predicted and $\mathrm{FEV}_{1} / \mathrm{FVC}<70 \%$. Particularly periodontitis and DMF-T were mainly linked to age and smoking.

As expected we found strong correlations between age and dental health, particularly regarding periodontitis and the composite DMF-T index. There was also a consistent association with smoking habits, in line with the literature $[18,19]$. In contrast, no significant associations between periodontitis and spirometric measures were present after adjustment for covariates. Recently, Holfreter and colleagues [10] found that measures of periodontitis were significantly linked to nearly all dynamic and static lung function indices. However, these were retrospective analyses, and dental health and lung function were assessed at different time points. The associations were clearly weaker when smoking was included as covariate. Moreover, the adjustment for body height reduced the correlations of periodontitis with lung function considerably. Therefore authors hypothesized that smaller persons might have a higher susceptibility to develop severe periodontitis. However, their findings could also be interpreted in the way that the use of \% predicted values of lung function, correcting for age, gender and height for each individual index, probably would have resulted in less strong associations.

In the present analysis we also explored the associations between other dimensions of dental health beyond periodontitis. Most spirometric indices expressed in absolute values were significantly different with regard to specific characteristics of dental health (carious lesions, dentures, missing teeth, oral mucosa disease, calculus). Using \% predicted values, dentures, missing teeth, oral mucosa diseases and a DMF-T-index > median were associated with lower $\mathrm{MEF}_{25}$ only. In further adjusted multivariate analyses, accounting for the difference between groups, only dentures were significantly linked to $\mathrm{MEF}_{25} \%$ predicted. Considering a potential causal relationship between reduced lung function and impaired dental health, it seems pathophysiologically plausible that the association is particularly reflected in the small airways where the initial changes in obstructive respiratory diseases occur [31]. Interestingly, Bergström and colleagues [18] recently found a weak but significant correlation between the occurrence of emphysema in $\mathrm{CT}$ scan or reduced carbon monoxide diffusing capacity and pocket depth and/or teeth loss. They hypothesized that this points towards an association between dental health and lung pathology not reflected in spirometry. Unfortunately they did not assess $\mathrm{MEF}_{50}$ or $\mathrm{MEF}_{25}$, which are capable of detecting minor spirometric airway abnormalities.

The association between dentures and lung function has been described in the latest literature by Linuma and co-workers [6]. Some authors described associations between missing or remaining teeth and lung function $[6,10]$ or the presence of COPD [12, 18, 22, 23]; these associations were not significant in our analysis after adjustment for covariates. From a dentist's perspective, both findings are not unexpected as dentures or missing teeth often reflect poor overall dental health status. As known, carious lesions acquired in early childhood or dentistry phobia often leads to early tooth loss and dentures; this phenomena is particularly observed in socially disadvantaged families [32-34]. This also fits to studies which found a link between oral hygiene status and COPD [11, 24, 35]. Surprisingly, the introduction of the composite DMF-T index did not enhance the associations in the present analysis, although patients with a higher DMF-T-index showed worse $\mathrm{MEF}_{25} \%$ predicted values. The associations probably have been weakened by the "F" (=fillings) subscore, which reflects a restored condition and not so much an inflammatory focus potentially linked to lung function impairment. In line with this, patients with dental fillings did not have worse lung function.

Oral mucosal disease was also associated with $\mathrm{MEF}_{25} \%$ predicted, but this association again was not significant after adjustment. This was most likely to the fact that the overall number of patients having oral mucosal diseases was rather low. In a larger population, gingival index was associated with airway obstruction in former smokers [21]. Moreover, a significant inverse correlation between gingival index and $\mathrm{FEV}_{1} \%$ predicted was recently reported in patients with COPD after adjustment for covariates [11]. 
Only few authors have considered radiologic abnormalities assessed by orthopantomogramms with regard to lung function; these studies addressed alveolar bone loss with regard to the presence of COPD. In the investigation by Wang and co-workers [22], the higher alveolar bone loss in COPD marginally failed statistical significance in univariate analysis, whereas in another study [12] it was very well associated with COPD. Similarly, Leukfeld and colleagues [14] observed that a mean marginal bone level $\geq 4 \mathrm{~mm}$ was associated with COPD independent of other risk factors for periodontitis. We found radiologic abnormalities in $47.9 \%$ of subjects but no significant associations with lung function. Similar results were obtained when including patients with alveolar bone loss of $\geq 1$ only (41.3\%). Possibly, radiologic abnormalities only occur in very advanced respiratory disease [14], but not in a general population as studied by us.

\section{Limitations}

An obvious limitation is the relatively low number of study participants. Many patients were not interested in lung function testing, medical research, or could not perform spirometry adequately. However, we consider it likely that this happened randomly and that we still investigated a representative sample. Since the study was performed in a dentist's practise, we had to dispense from postbronchodilator measurements. Thus, the presence of COPD via $\mathrm{FEV}_{1} /$ FVC $<70 \%$ might have been overestimated. $\mathrm{MEF}_{50}$ and $\mathrm{MEF}_{25}$ could not be determined in all patients, however a full determination would presumably enhance the significance of the associations. As the study is observational, it does not allow inferring causal relationships. The determination of serum inflammatory markers or oral bacterial colonization would have been desirable regarding potential interactions between lung and dental health, although in a recent analysis fibrinogen and high-sensitive $\mathrm{C}$-reactive were not suggestive of links between systemic inflammation and lung function [10]. We characterised patients comprehensively with regard to dental health, but the protocol had to remain feasible and we might have missed some factors. Actually, tooth brushing times and method, experience of dental floss use, dental visits and oral health knowledge have been demonstrated to be significantly related to the risk of COPD [14, 22]. We could not adjust the analysis for these factors, similarly as, e.g., Holfreter and collegues in their impressive analysis [10]. Whether the inclusion of the profession (academic versus non-academic) or school education [10] is sufficient to account for these factors, is unclear.

\section{Conclusion}

In conclusion, this prospective cross-sectional study assessed the associations between dental health and spirometric measures in a general real-life population, using a broad panel of dental health measures. The major result was that dentures, missing teeth, oral mucosa diseases and a higher DMFT were weakly linked to impaired age-adjusted indices of small airways dysfunction in terms of $\mathrm{MEF}_{25} \%$ predicted, while dentures were the only dimension remaining significant in multivariate analyses. Our data suggest that at least phenomenologically, the link between lung function and dental health is weak, compared to the needs for relevant predictions in individuals. Further prospective studies could benefit from the information on specific assessments of dental health provided by our study. These studies might also address respiratory disorders beyond the diagnosis of COPD, particularly with regard to small airway dysfunction.

\section{Abbreviations \\ BMI: body-mass-index; NYHA: New York Heart Association; FEV 1 : forced expira- tory volume in $1 \mathrm{~s}$; FVC: forced vital capacity; PEF: peak expiratory flow; $\mathrm{MEF}_{50}$ : maximal expiratory flow at $50 \%$ of vital capacity; $\mathrm{MEF}_{25}$ : maximal expiratory flow at $25 \%$ of vital capacity; GLI: reference values according to global lungs initiative; DMF-T: decayed-missed-filled-teeth-index; COPD: chronic obstruc- tive pulmonary disease; PSI: periodontal screening-index; ATS: American Thoracic Society; PEF: peak expiratory flow; LLN: lower limit of normal; WHO: World Health Organization; PD: probing depth; S1-S3: sextants maxillary (S1- S3, right-posterior, front, left-posterior); S4-S6: sextants mandibular (S4-S6, left- posterior, front, right-posterior); GOLD: global Initiative for chronic obstructive Lung disease; NHANES: National Health and Nutrition Examination Survey; CAL: clinical attachment loss; CT: computed tomography; CPITN: community periodontal index of treatment needs.}

\section{Authors' contributions}

The contributions of the authors are as follows. $\mathrm{CH}$ collected the data, participated in the design of the study, participated in the statistical evaluation and helped in drafting and writing the manuscript. RAJ participated in the statistical evaluation of the data, their interpretation and in writing the manuscript. SB carried out and designed the study, performed part of the data evaluation and drafted the manuscript. All authors had full access to all the data in the study and take responsibility for the integrity of the data and the accuracy of the analysis. All authors read and approved the final manuscript.

\section{Author details}

${ }^{1}$ General Dental Practice, Mühldorf, Germany. ${ }^{2}$ Department of Internal Medicine III, Division of Pulmonary and Respiratory Medicine, RoMed Clinical Centre, Rosenheim, Germany. ${ }^{3}$ Institute and Outpatient Clinic for Occupational, Social and Environmental Medicine, Ludwig-Maximilians-University, Munich, Germany.

\section{Competing interests}

The authors declare that they have no competing interests.

Received: 21 December 2015 Accepted: 6 May 2016

Published online: 17 May 2016

\section{References}

1. Mannino DM, Buist AS. Global burden of COPD: risk factors, prevalence, and future trends. Lancet. 2007:370:765-73.

2. Buist AS, McBurnie MA, Vollmer WM, Gillespie S, Burney P, Mannino DM, Menezes AMB, Sullivan SD, Lee TA, Weiss KB, Jensen RL, Marks GB, Gulsvik A, Nizankowska-Mogilnicka E. International variation in the prevalence of COPD (the BOLD Study): a population-based prevalence study. Lancet. 2007:370:741-50. 
3. Menezes AMB, Perez-Padilla R, Jardim JRB, Muiño A, Lopez MV, Valdivia G, de Montes Oca M, Talamo C, Hallal PC, Victora CG. Chronic obstructive pulmonary disease in five Latin American cities (the PLATINO study): a prevalence study. Lancet. 2005;366:1875-81.

4. Salvi SS, Barnes PJ. Chronic obstructive pulmonary disease in non-smokers. Lancet. 2009;374:733-43.

5. Cullinan MP, Ford PJ, Seymour GJ. Periodontal disease and systemic health: current status. Aust Dent J. 2009;54(Suppl 1):62-9.

6. Linuma T, Arai Y, Abe Y, Takayama M, Fukumoto M, Fukui Y, Iwase T, Takebayashi T, Hirose N, Gionhaku N, Komiyama K. Denture wearing during sleep doubles the risk of pneumonia in the very elderly. J Dent Res. 2015;94(3 Suppl):28-36.

7. Scannapieco FA, Papandonatos GD, Dunford RG. Associations between oral conditions and respiratory disease in a national sample survey population. Ann Periodontol. 1998;3:251-6.

8. Didilescu AC, Skaug N, Marica C. Respiratory pathogens in dental plaque of hospitalized patients with chronic lung diseases. Clin Oral Investig. 2005;9:141-7

9. Tonetti MS, D'Aiuto F, Nibali L, Donald A, Storry C, Parkar M, Suvan J, Hingorani AD, Vallance P, Deanfield J. Treatment of periodontitis and endothelial function. N Engl J Med. 2007;356:911-20.

10. Holtfreter B, Richter S, Kocher T, Dörr M, Völzke H, Ittermann T, Obst A Schäper C, John U, Meisel P, Grotevendt A, Felix SB, Ewert R, Gläser S. Periodontitis is related to lung volumes and airflow limitation - a crosssectional study. Eur Respir J. 2012. (Epub ahead of print).

11. Peter KP, Mute BR, Doiphode SS, Bardapurkar SJ, Borkar MS, Raje DV. Association between periodontal disease and chronic obstructive pulmonary disease-a reality or just a dogma. J Periodontol. 2013. [Epub ahead of print].

12. Si Y, Fan H, Song Y, Zhou X, Zhang J, Wang Z. Association between periodontitis and chronic obstructive pulmonary disease in a Chinese population. J Periodontol. 2012;83:1288-96.

13. Kucukcoskun M, Baser U, Oztekin G, Kiyan E, Yalcin F. Initial periodontal treatment for prevention of chronic obstructive pulmonary disease exacerbations. J Periodontol. 2013;84:863-70.

14. Leuckfeld I, Obregon-Whittle MV, Lund MB, Geiran O, Bjørtuft $\varnothing$, Olsen I. Severe chronic obstructive pulmonary disease: association with marginal bone loss in periodontitis. Respir Med. 2008;102:488-94.

15. Deo V, Bhongade ML, Ansari S, Chavan RS. Periodontitis as a potential risk factor for chronic obstructive pulmonary disease: a retrospective study. Indian J Dent Res. 2009;20:466-70.

16. Scannapieco FA, Ho AW. Potential associations between chronic respiratory disease and periodontal disease: analysis of National Health and Nutrition Examination Survey III. J Periodontol. 2001;72(1):50.

17. Offenbacher S, Beck JD, Barros SP, Suruki RY, Loewy ZG. Obstructive airway disease and edentulism in the atherosclerosis risk in communities (ARIC) study. BMJ Open. 2012;2(6):e001615.

18. Bergström J, Cederlund K, Dahlén B, Lantz A, Skedinger M, Palmberg L, Sundblad B, Larsson K. Dental health in smokers with and without COPD. PLoS One. 2013;8:e59492.

19. Hyman JJ, Reid BC. Cigarette smoking, periodontal disease: and chronic obstructive pulmonary disease. J Periodontol. 2004;75:9-15.
20. Zeng X, Tu M, Liu D, Zheng D, Zhang J, Leng W. Periodontal disease and risk of chronic obstructive pulmonary disease: a meta-analysis of observational studies. PLoS One. 2012;7:e46508.

21. Katancik JA, Kritchevsky S, Weyant RJ, Corby P, Bretz W, Crapo RO, Jensen R, Waterer G, Rubin SM, Newman AB. Periodontitis and airway obstruction. J Periodontol. 2005;76:2161-7.

22. Wang Z, Zhou X, Zhang J, Zhang L, Song Y, Hu FB, Wang C. Periodontal health, oral health behaviours, and chronic obstructive pulmonary disease. J Clin Periodontol. 2009;36:750-5.

23. Zhou X, Han J, Song Y, Zhang J, Wang Z. Serum levels of 25-hydroxyvitamin $D$, oral health and chronic obstructive pulmonary disease. J Clin Periodontol. 2012;39:350-6.

24. Sharma N, Shamsuddin H. Association between respiratory disease in hospitalized patients and periodontal disease: a cross-sectional study. J Periodontol. 2011;82:1155-60.

25. Klein H, Palmer CE, Knutson JW. Studies on dental caries. Dental status and dental needs of elementary school children. Public Health Rep. 1938:53:751-65.

26. American Thoracic Society 1995. Standardization of Spirometry. Update Am J Respir Crit Care Med. 1994;152(Suppl 3):1107-36.

27. Quanjer PH, Tammeling GJ, Cotes JE, Pedersen OF, Peslin R, Yernault JC. Lung volumes and forced ventilatory flows. Report working party standardization of lung function tests, european community for steel and coal. Official statement of the European Respiratory Society. Eur Respir J Suppl. 1993;16:5-40.

28. Quanjer PH, Hall GL, Stanojevic S, Cole TJ, Stocks J. Age- and heightbased prediction bias in spirometry reference equations. Eur Respir J. 2012:40:190-7

29. ADA and AAP introduce dentists to new time saving periodontal evaluation system. Va Dent J. 1992. 69(Suppl 4):16-17.

30. Henke C, Jörres R, Budweiser S. Lung function and associations with multiple dimensions of dental health. ERJ. 2014;44:803.

31. McDonough JE, Yuan R, Suzuki M, Seyednejad N, Elliott WM, Sanchez PG, Wright AC, Gefter WB, Litzky L, Coxson HO, Paré PD, Sin DD, Pierce RA, Woods JC, McWilliams AM, Mayo JR, Lam SC, Cooper JD, Hogg JC. Smallairway obstruction and emphysema in chronic obstructive pulmonary disease. N Engl J Med. 2011;365:1567-75.

32. Holst D, Schuller AA. Oral health in a life-course: birth-cohorts from 1929 to 2006 in Norway. Community Dent Health. 2012;29:134-43.

33. Riley JL, Gilbert GH. Childhood dental history and adult dental attitudes and beliefs. Int Dent J. 2005;55:142-50

34. Pearce MS, Steele JG, Mason J, Walls AWG, Parker L. Do circumstances in early life contribute to tooth retention in middle age. J Dent Res. 2004:83:562-6.

35. Liu Z, Zhang W, Zhang J, Zhou X, Zhang L, Song Y, Wang Z. Oral hygiene, periodontal health and chronic obstructive pulmonary disease exacerbations. J Clin Periodontol. 2012;39:45-52.

\section{Submit your next manuscript to BioMed Central and we will help you at every step:}

- We accept pre-submission inquiries

- Our selector tool helps you to find the most relevant journal

- We provide round the clock customer support

- Convenient online submission

- Thorough peer review

- Inclusion in PubMed and all major indexing services

- Maximum visibility for your research

Submit your manuscript at www.biomedcentral com/submit 